\title{
New somatic BRAF splicing mutation in Langerhans cell histiocytosis
}

\author{
Sébastien Héritier ${ }^{1,2,3^{*}+}$, Zofia Hélias-Rodzewicz ${ }^{2,4 \dagger}$, Rikhia Chakraborty ${ }^{5,6 \dagger}$, Amel G. Sengal ${ }^{5,6}$, \\ Christine Bellanné-Chantelot ${ }^{7}$, Caroline Thomas ${ }^{8}$, Anne Moreau ${ }^{9}$, Sylvie Fraitag ${ }^{10}$, Carl E. Allen ${ }^{5,6}$, \\ Jean Donadieu ${ }^{1,2,3+}$ and Jean-François Emile 2,4 $^{2}$
}

\begin{abstract}
Langerhans cell histiocytosis $(\mathrm{LCH})$ is an inflammatory myeloid neoplasia with constitutive activation of the MAPKinase RAS-RAF-MEK-ERK cell signaling pathway. We analyzed 9 LCH cases without BRAF ${ }^{V 600}$ and MAP2K1 mutations by whole exome sequencing. We identified a new somatic BRAF splicing mutation in 2 cases. Both cases were childhood single system (SS) LCH cases, with self-healing outcome of the bone lesions. This mutant consisted in a 9 base pair duplication (c.1511_1517 + 2 duplication), encoding for a predicted mutant protein with insertion of 3 amino acids (p.Arg506_Lys507insLeuLeuArg) in the N-terminal lobe of the kinase domain of BRAF. Transient expression of the c.1511_1517 + 2dup BRAF mutant in HEK293 cells enhanced MAPKinase pathway activation, and was not inhibited by vemurafenib but was inhibited by PLX8394, a second-generation BRAF inhibitor able to inhibit signaling of BRAF monomers and dimers. Future LCH molecular screening panel should include this new mutation to better define its prevalence in LCH and its restriction to autoregressive bone SS LCH.
\end{abstract}

Keywords: Langerhans cell histiocytosis, BRAF, Splicing mutation, Targeted therapy

\section{Background}

Langerhans cell histiocytosis ( $\mathrm{LCH})$ is the most common histiocytosis, and is characterized by inflammatory lesions containing abundant CD1a + CD207+ histiocytes that lead to the destruction of affected tissues [1]. A $B R A F^{\mathrm{V} 600 \mathrm{E}}$ mutation, responsible for activation of the MAPKinase RAS-RAF-MEK-ERK cell signaling pathway in pathologic histiocytes, is present in $\sim 55 \%$ of $\mathrm{LCH}$ cases and was associated with recurrence and high-risk presentation [2]. Responses to BRAF inhibitors in patients with $B R A F^{\mathrm{V} 600 \mathrm{E}}$-mutated LCH confirms that $B R A F^{\mathrm{V} 600 \mathrm{E}}$ is a driver mutation in $\mathrm{LCH}$ [3]. Although $\sim 45 \%$ do not have $B R A F^{\mathrm{V} 600 \mathrm{E}}$ mutation, ERK was reported to be activated in pathologic histiocytes of all LCH samples [4]. Other molecular alterations have also been reported to activate the MAPKinase pathway in $B R A F^{\mathrm{V} 600 \mathrm{E}}$-non $\mathrm{mu}$ tated $\mathrm{LCH}$, such as $M A P 2 K 1$ mutations $(10-20 \%$ of $\mathrm{LCH})[5,6], \beta 3-\alpha \mathrm{C}$ loop deletion in the kinase domain of BRAF (6\% of $\mathrm{LCH})$ [7], and case reports

\footnotetext{
*Correspondence: sebastien.heritier@aphp.fr

${ }^{\dagger}$ Equal contributors

${ }^{1}$ French Reference Center for Langerhans Cell Histiocytosis, Trousseau Hospital, Assistance Publique-Hôpitaux de Paris, Paris, France

${ }^{2}$ EA4340, Versailles SQY University, Paris-Saclay University, Boulogne, France Full list of author information is available at the end of the article
}

highlighted mutation on $A R A F$ [8] and MAP3K1 [9]. Fusion events involving $B R A F$ and activating MAPkinase pathway have also been reported in histiocytoses of the $\mathrm{L}$ group $[7,10]$.

To identify the mechanism of pathologic ERK activation in the remaining $\mathrm{LCH}$, we performed whole exome sequencing (WES) on selected LCH frozen biopsy samples wild-type for the most common activating mutations reported in LCH. DNA extracted from peripheral white blood cells (PBMC) were used as the "normal" sample for comparison. Mutation function and response to MAPKinase pathway inhibitors were assessed using in vitro constructs.

\section{Results}

From the French LCH registry [11], 9 patients fulfilled the following inclusion criteria: i) fresh frozen biopsy tissue and blood samples available, ii) high percentage of lesionsinfiltrating CD207+ histiocytes ( $>30 \%$ ), iii) no mutation identified by $B R A F^{\mathrm{V} 600 \mathrm{E}}$ pyrosequencing [2] or among the most common activating mutations of PIK3CA, BRAF, $K R A S$ and NRAS with the i-plex mass spectrometric based genotyping technology (Sequenom-Agena Bioscience) [12], iv) negative screening for exon 2-3 MAP2K1 mutations by 
Sanger sequencing. Among the 9 included patients, 7 had a bone-limited $\mathrm{LCH}$ and 2 had a $\mathrm{LCH}$ involving several organs (Table 1).

\section{Detection of duplication at the end of BRAF exon 12 in LCH samples}

A somatic duplication of 9 base pairs at the end of exon 12 of BRAF (nucleotides c.1511_1517 + 2) was detected in $\mathrm{LCH}$ samples from 2 patients (P5 and P6). Both patients were children with self-healing bone lesions. This duplication was not yet reported in the COSMIC database. For both patients, Sanger sequencing of genomic DNA confirmed the $B R A F$ c.1511_1517 + 2 duplication in LCH lesions (Fig. 1a), but failed to detect it within PBMC. This 9 nucleotides insertion at the position +2 of the splice donor site of intron 12 was predicted to change the splicing, with an insertion of 9 nucleotides in the cDNA sequence [GTTACTCAG] at the end of exon 12 (Fig. 1b). Messenger RNA was extracted from lesion of P5, and length analysis of PCR products of cDNA confirmed a 9 nucleotides insertion (Fig. 1c). Insertion was also confirmed by Sanger sequencing (Additional file 1: Figure. S1).

To investigate the prevalence of the somatic $B R A F$ c.1511_1517 + 2 duplication in LCH, we studied 28 additional LCH samples wild-type for $B R A F^{\mathrm{V} 600}$, by length analysis of PCR products. No additional mutated case was found, suggesting that this mutation represents a small proportion of $B R A F^{\mathrm{V} 600}$ wild-type $\mathrm{LCH} \quad(<10 \%)$ [7], but more studies are needed to estimate precisely its prevalence.

Functional analysis and response to MAPkinase inhibitors The insertion of 3 amino acid (p.Arg506_Lys507insLeu LeuArg) coded by this 9 base pair duplication is localized in the smaller $\mathrm{N}$-terminal lobe of the kinase BRAF domain responsible for ATP binding (Additional file 1: Figure. S2). Small deletions localized nearby this region of $B R A F$ were shown to induce MAPkinase pathway activation in $\mathrm{LCH}$ and in pancreatic carcinomas $[7,13]$, suggesting that this new mutation may also have functional impact. Immunohistochemistry of samples of P5 and P6 confirmed that areas rich in CD1a + histiocytes contained numerous histiocytes with phosphoERK in their cytoplasms as well as translocated into the nucleus (Fig. 1d). The strong phosphorylation of ERK in LCH lesions of P5 and P6 was also confirmed by Western blot (Fig. 1e).

We then assessed the functional impact of this genomic alteration on $B R A F$ signaling by analyzing phosphorylation of ERK in HEK293 cells transiently transfected with wild-type $B R A F, B R A F^{V 600 E}$ or BRAF c.1511_1517 + 2dup mutants. cDNA expression of BRAF c.1511_1517 + 2dup, but not wild-type $B R A F$, resulted in a significant increase in ERK1/2 phosphorylation (Fig. 1f).

We also evaluated the ability of the BRAF ${ }^{\mathrm{V} 600 \mathrm{E}}$ inhibitor vemurafenib and the MEK inhibitor trametinib to suppress ERK activation by specific $B R A F$ alterations. Although vemurafenib induced a substantial inhibition of $B R A F^{V 600 E}$-induced activation, this drug did not inhibit the MAPkinase activation in cells transfected with the cDNA containing the BRAF c.1511_1517 + 2dup mutant, which is consistent with specific activity of this agent against mutations that result in active BRAF monomers. Trametinib, which blocks active MEK, decreased activation of ERK in cells transfected with $B R A F^{\mathrm{V} 600 \mathrm{E}}$, but with no impact on cells transfected with the BRAF c.1511_1517 + 2dup mutant (Fig. 1f). We thus further evaluated the effects of other, or combination of inhibitors of the MAPkinase pathway. As expected TCS ERK 11e, which directly inhibits ERK, induced a total extinction of ERK phosphorylation (Fig. 1g). PLX8394 is a second-generation BRAF inhibitor able to inhibit signaling of BRAF monomers and dimers without paradoxical activation of MAPKpathway signaling in cells with wild-type BRAF that has been observed in firstgeneration agents such as vemurafenib [14, 15]. PLX8394 induced an almost complete extinction of pERK signal on Western blot, confirming that most of the pathway activation was due to the mutant $B R A F$. Again vemurafenib or trametinib alone did not suppress ERK activation, but combination of both drugs induced a completed extinction of the pERK signal (Fig. 1g).

To elucidate this last observation, we performed a dose response experiment with vemurafenib and trametinib on BRAF c.1511_1517 + 2dup transfected cells and $B R A F^{\mathrm{V} 600 \mathrm{E}}$ transfected cells, in order to test if an increased dose for trametinib was required to block activation by the BRAF c.1511_1517 + 2dup mutation as compared to other $\mathrm{LCH}$-associated $B R A F$ mutations. In our model, while vemurafenib and trametinib induced an inhibition of $B R A F^{V 600 E}$-induced activation with relationship between dose and response, these drugs did not inhibit the MAPkinase activation in BRAF c.1511_1517 + 2dup transfected cells regardless of dose (Fig. 1h). Neither vemurafenib nor trametinib used individually, even at the highest concentration, could inhibit phosphorylated MEK1/2 and phosphorylated ERK1/2. Future study should better define mechanisms of resistance of the BRAF c.1511_1517 + 2dup mutation by targeted therapies such as vemurafenib and trametinib.

\section{Conclusions}

We report here a new somatic $B R A F$ splicing mutation in $\mathrm{LCH}$, leading to the insertion of 3 amino acids (p.Arg506_Lys507insLeuLeuArg) in the N-terminal lobe of the kinase domain of BRAF. This mutation constitutively 


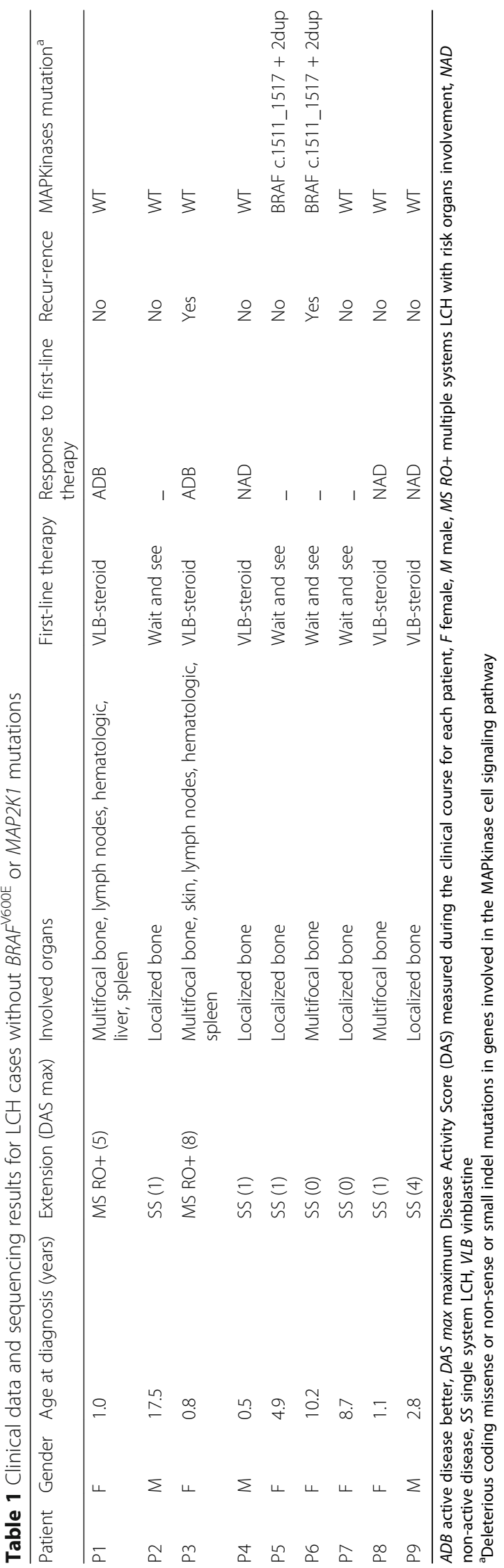




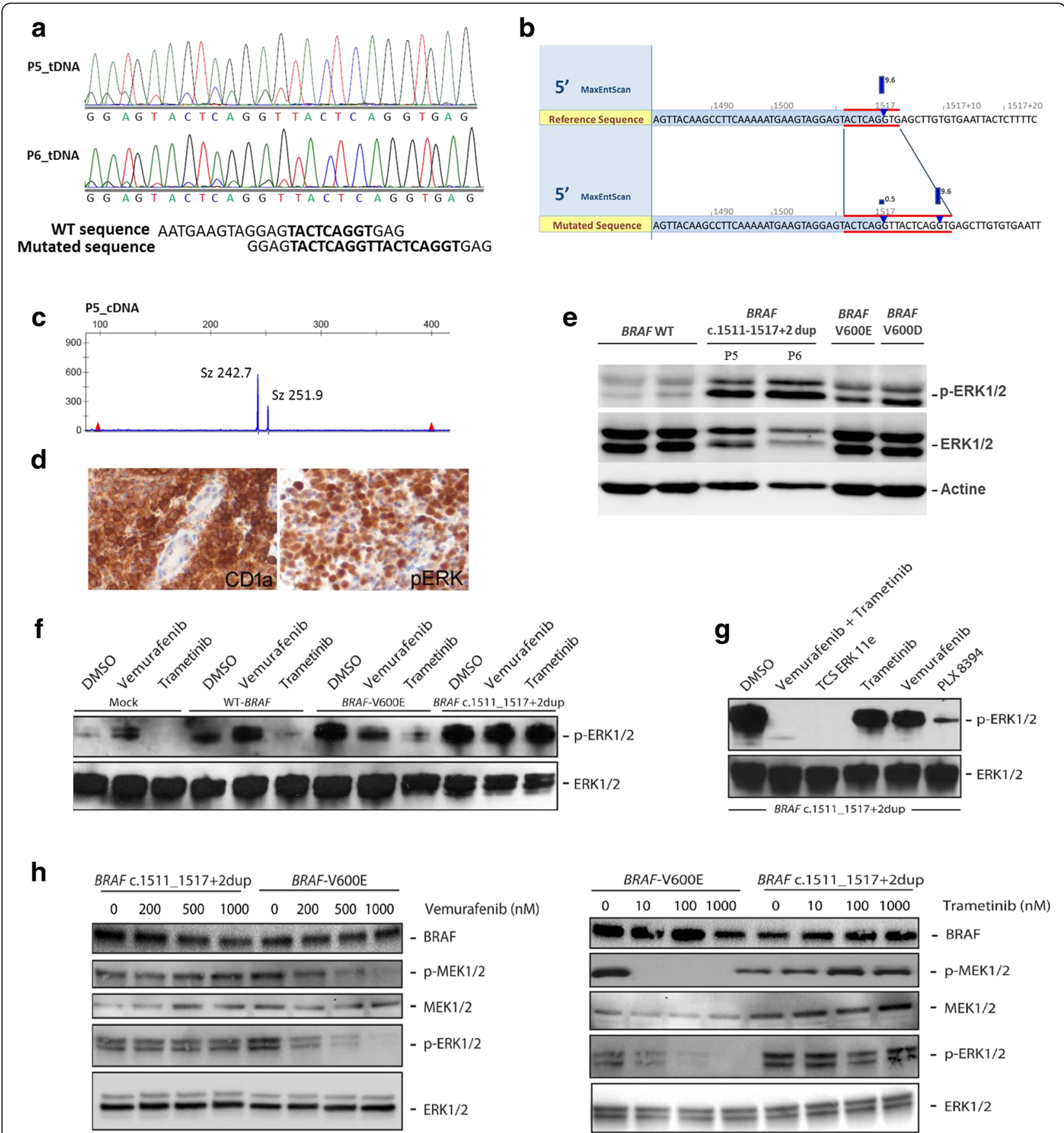

Fig. 1 Analysis of LCH samples. a Sanger sequencing of P5 and P6 LCH samples shows duplication of the c.1511_1517+ 2 sequence. b In silico analysis (Alamut ${ }^{\oplus}$ Visual, hg19) predicts a $5^{\prime}$ splice site change, causing the insertion of 9 nucleotides in the CDNA sequence [GTTACTCAG] at the end of exon 12. (C) P5 CDNA analyse confirms insertion of 9 nucleotides by rt.-PCR product length analysis. $\mathbf{d}$ Immunohistochemistry performed on FFPE samples from P5 showed a strong cytoplasmic and nuclear positivity of histiocytes with phosphoERK1/2 (D13.14.4E, Rabbit mAb, Cell Signaling) in areas containing numerous CD1a + LCH cells. e Results of the western blot ( $p$ - and total-ERK1/2) for P5 and P6 LCH. Protein extracts from two $B R A F$ wild type, a $B R A F^{\mathrm{V} 600 \mathrm{E}}$-mutated $\mathrm{LCH}$ and a $B R A F^{\mathrm{V} 600 \mathrm{D}}$-mutated $\mathrm{LCH}$ were used as positive control for $\mathrm{p}$-ERK. Functional analysis of the BRAF c.1511_1517 + 2 duplication. HEK293 cells were transiently transfected with expression plasmids encoding BRAF wild-type, BRAF ${ }^{V 600 E}$ and BRAF c.1511_1517 + 2dup mutant CDNAs, and corresponding lysates from cells maintained in serum were subjected to immunoblotting with the indicated antibodies. $\mathbf{f}$ Where indicated, cells were treated with inhibitor of BRAF ${ }^{\mathrm{V} 600 \mathrm{E}}$ (vemurafenib) or MEK (trametinib) for $4 \mathrm{~h}$ before harvest. $\mathbf{g}$ Where indicated, cells were treated with combination of vemurafenib and trametinib, or with inhibitors of BRAF (PLX8394) or ERK (TCS ERK 11e) for $4 \mathrm{~h}$ before harvest. $\mathbf{h}$ To test dose response to vemurafenib and trametinib on BRAF ${ }^{V 600 E}$ and BRAF c.1511_1517 + 2dup transfected cells, the cells were treated for $4 \mathrm{~h}$ with the specified agents (vemurafenib or trametinib) at the specified doses before harvest 
activates the MAPKinase pathway, and was inhibited by the second-generation BRAF inhibitor PLX8394. Thanks to recent substantial effort of LCH expert teams, the unknown part of the molecular spectrum of $\mathrm{LCH}$ continues to shrink and identification of these mutations has many potential applications such as targeted therapy, therapeutic riskstratification based on tumor genotype, and quantitative detection of mutant allele in circulating cell free DNA as possible blood biomarkers.

\section{Additional file}

Additional file 1: Supplemental methods and data. (DOCX 904 kb)

\section{Abbreviations}

ADB: Active disease better; DAS: Disease activity score; F: Female; LCH: Langerhans cell histiocytosis; M: Male; MS RO+ LCH: Multiple systems LCH with risk organ involvement; NAD: Non-active disease; PBMC: Peripheral white blood cells; SS LCH: Single system LCH; VLB: Vinblastine; WES: Whole exome sequencing

\section{Acknowledgements}

We thank the patients and their families for their participation in this study. The authors thank Mr. M. Barkaoui and Mr. J. Miron who collected clinical data. We would also like to thank Gideon Bollag and the Plexxikon team for helpful discussions, collaboration and access to PLX8394.

\section{Funding}

This study received grants from the Société Française de lutte contre les Cancers de l'Enfant et de l'Adolescent, the Fédération Enfants et Santé, the Association Recherche et Maladie Hématologiques de l'Enfant; the Association Les 111 des Arts de Paris; the Association la Petite Maison dans la Prairie; the Association pour la Recherche et l'Enseignement en Pathologie (AREP), and from the Gardrat family. This project received constant, unlimited support from the Association Histiocytose France. The French LCH registry was supported by a grant from InVS and INSERM for the rare disease registry and a grant from Roche.

\section{Availability of data and materials}

The datasets used and/or analyzed during the current study are available from the corresponding author on reasonable request.

\section{Authors' contributions \\ SH, JD and JFE conceived the study. SH, ZHR, RC, AGS, CEA, JD and JFE designed the experiments. SH, ZHR, RC and AGS performed research. SH, ZHR, RC, AGS, CEA, CBC, JD and JFE analyzed and interpreted data; CT, AM and SF contributed to samples and clinical information collection. $\mathrm{SH}, \mathrm{ZHR}$, RC, CEA, JD and JFE drafted the manuscript. All authors read and approved the final manuscript.}

\section{Ethics approval and consent to participate}

This study was approved by the ethics committee Ile de France III (\#2011A00447-34) and conducted in accordance with the Declaration of Helsinki.

\section{Consent for publication}

Not applicable.

\section{Competing interests}

JFE received honoraria from Roche, GlaxoSmithKline (GSK) and Pierre Fabre. The remaining authors declare no competing financial interests.

\section{Publisher's Note}

Springer Nature remains neutral with regard to jurisdictional claims in published maps and institutional affiliations.

\section{Author details}

${ }^{1}$ French Reference Center for Langerhans Cell Histiocytosis, Trousseau Hospital, Assistance Publique-Hôpitaux de Paris, Paris, France. ²EA4340, Versailles SQY University, Paris-Saclay University, Boulogne, France.

${ }^{3}$ Department of Pediatric Hematology and Oncology, Trousseau Hospital, Assistance Publique-Hôpitaux de Paris, Paris, France. ${ }^{4}$ Pathology Department, Ambroise Paré Hospital, Assistance Publique-Hôpitaux de Paris, Boulogne, France. ${ }^{5}$ Texas Children's Cancer Center, Texas Children's Hospital, Houston, TX, USA. ${ }^{6}$ Division of Pediatric Hematology-Oncology, Department of Pediatrics, Baylor College of Medicine, Houston, TX, USA. ${ }^{7}$ Department of Genetics, Pitié-Salpétrière Hospital, Assistance Publique-Hôpitaux de Paris, Paris, France. ${ }^{8}$ Department of Pediatric Hematology and Oncology, Centre Hospitalo-Universitaire de Nantes, Nantes, France. ${ }^{9}$ Pathology Department, Centre Hospitalo-Universitaire de Nantes, Nantes, France. ${ }^{10}$ Pathology Department, Necker Hospital, Assistance Publique-Hôpitaux de Paris, Paris, France.

Received: 10 March 2017 Accepted: 28 June 2017

Published online: 06 July 2017

\section{References}

1. Emile J-F, Abla O, Fraitag S, Horne A, Haroche J, Donadieu J, et al. Revised classification of histiocytoses and neoplasms of the macrophage-dendritic cell lineages. Blood. 2016;127:2672-81.

2. Héritier S, Emile J-F, Barkaoui M-A, Thomas C, Fraitag S, Boudjemaa S, et al. BRAF Mutation Correlates With High-Risk Langerhans Cell Histiocytosis and Increased Resistance to First-Line Therapy. J Clin Oncol. 2016;34:3023-30.

3. Héritier S, Jehanne M, Leverger G, Emile J-F, Alvarez J-C, Haroche J, et al. Vemurafenib Use in an Infant for High-Risk Langerhans Cell Histiocytosis. JAMA Oncol. 2015;1:836-8.

4. Badalian-Very G, Vergilio J-A, Degar BA, MacConaill LE, Brandner B, Calicchio ML, et al. Recurrent BRAF mutations in Langerhans cell histiocytosis. Blood. 2010;116:1919-23.

5. Brown NA, Furtado LV, Betz BL, Kiel MJ, Weigelin HC, Lim MS, et al. High prevalence of somatic MAP2K1 mutations in BRAF V600E-negative Langerhans cell histiocytosis. Blood. 2014;124:1655-8.

6. Chakraborty R, Hampton OA, Shen X, Simko SJ, Shih A, Abhyankar H, et al. Mutually exclusive recurrent somatic mutations in MAP2K1 and BRAF support a central role for ERK activation in LCH pathogenesis. Blood. 2014;124:3007-15

7. Chakraborty R, Burke TM, Hampton OA, Zinn DJ, Lim KPH, Abhyankar H, et al. Alternative genetic mechanisms of BRAF activation in Langerhans cell histiocytosis. Blood. 2016;128:2533-7.

8. Nelson DS, Quispel W, Badalian-Very G, van Halteren AGS, van den Bos C, Bovée JVMG, et al. Somatic activating ARAF mutations in Langerhans cell histiocytosis. Blood. 2014;123:3152-5.

9. Nelson DS, van Halteren A, Quispel WT, van den Bos C, Bovée JVMG, Patel $\mathrm{B}$, et al. MAP2K1 and MAP3K1 mutations in Langerhans cell histiocytosis. Genes Chromosomes Cancer. 2015;54:361-8.

10. Diamond EL, Durham BH, Haroche J, Yao Z, Ma J, Parikh SA, et al. Diverse and Targetable Kinase Alterations Drive Histiocytic Neoplasms. Cancer Discov. 2015;6(2):154-65.

11. Rigaud C, Barkaoui MA, Thomas C, Bertrand Y, Lambilliotte A, Miron J, et al. Langerhans cell histiocytosis: therapeutic strategy and outcome in a 30-year nationwide cohort of 1478 patients under 18 years of age. $\mathrm{Br} J$ Haematol. 2016;174:887-98.

12. Héritier S, Saffroy R, Radosevic-Robin N, Pothin Y, Pacquement H, Peuchmaur M, et al. Common cancer-associated PIK3CA activating mutations rarely occur in Langerhans cell histiocytosis. Blood. 2015;125:2448-9.

13. Foster SA, Whalen DM, Özen A, Wongchenko MJ, Yin J, Yen I, et al. Activation Mechanism of Oncogenic Deletion Mutations in BRAF, EGFR, and HER2. Cancer Cell. 2016;29:477-93.

14. Zhang C, Spevak W, Zhang Y, Burton EA, Ma Y, Habets G, et al. RAF inhibitors that evade paradoxical MAPK pathway activation. Nature. 2015:526:583-6

15. Karoulia Z, Wu Y, Ahmed TA, Xin Q, Bollard J, Krepler C, et al. An Integrated Model of RAF Inhibitor Action Predicts Inhibitor Activity against Oncogenic BRAF Signaling. Cancer Cell. 2016;30:485-98. 